\title{
Meeting Report: International Workshop on Implementation of Biowaivers Based on the Biopharmaceutics Classification System (BCS)
}

\author{
Rodrigo Cristofoletti ${ }^{1,7}$, Vinod P. Shah², Peter Langguth ${ }^{3}$, James E. Polli ${ }^{4}$, Mehul Mehta ${ }^{5}$, \\ Bertil Abrahamsson6, Jennifer B. Dressman7, and Pablo Quiroga8,* \\ ${ }^{1}$ Brazilian Health Surveillance Agency (Anvisa), Division of Bioequivalence, Brasilia, Brazil \\ ${ }^{2}$ International Pharmaceutical Federation, The Hague, The Netherlands \\ ${ }^{3}$ Department of Pharmaceutical Technology and Biopharmaceutics, Johannes Gutenberg University, Mainz, Germany \\ ${ }^{4}$ Department of Pharmaceutical Sciences, School of Pharmacy, University of Maryland, Baltimore, Maryland, USA \\ ${ }^{5}$ Food and Drug Administration, CDER \\ ${ }^{6}$ AstraZeneca R\&D, Mölndal, Sweden \\ ${ }^{7}$ Institute of Pharmaceutical Technology, J.W. Goethe University, Frankfurt am Main, Germany \\ ${ }^{8}$ National University of La Plata, La Plata, Argentina
}

This paper reflects the scientific opinion of the authors and not the policies of the regulatory agencies or the companies for which they work.

\section{INTRODUCTION}

E ven though the pivotal article stating the theoretical basis for a biopharmaceutics drug classification (1) was published almost 20 years ago, the extension of BCS-based biowaiver decisions to drugs belonging to BCS classes other than those showing high solubility and high permeability has not yet reached a consensus among regulators, industrial scientists, and academics. Also, within some jurisdictions, BCS principles have not yet been incorporated into legal frameworks and thus have not been used to allow science- and risk-based regulatory flexibility.

This report provides a brief description of the presentations from the International Workshop on Implementation of Biowaivers based on the BCS in Buenos Aires, Argentina, that took place on March 5-6, 2015. The meeting was cosponsored by National University of La Plata, Confederación Farmacéutica Argentina, International Pharmaceutical Federation (FIP), and the American Association of Pharmaceutical Scientists (AAPS). The main objectives of the meeting were to describe the state of the art with respect to in vitro and in silico tools to support waiving in vivo bioequivalence studies and to foster discussion about implementing BCS-based biowaiver decisions to support generic drug registration in South America. Two hundred and fifteen scientists from universities, the pharmaceutical industry, and regulatory authorities took part in this meeting.

\section{THE PRESENTATIONS}

The Chairs of the meeting, Prof. Pablo Quiroga, Dr. Rodrigo Cristofoletti, Prof. Jennifer Dressman, and the FIP CEO, Dr. Luc Besancon, opened the meeting and welcomed the audience and speakers.

In Plenary Session 1, Dr. Vinod Shah presented the scientific principles of BCS and how the Food and Drug Administration (FDA) incorporated them into its regulatory framework, firstly as an important tool for setting the requirements for supporting post-approval changes and, subsequently, for supporting new and abbreviated drug applications. Prof. Dr. Jennifer Dressman spoke about methods to characterize drug solubility according to BCS and pointed out some pitfalls found in the description of drug solubility assessment using shake-flask methods extracted from a CRO website. She also discussed the dose definition for drug solubility classification purposes. Using different dose definitions, "highest single therapeutic dose" or "highest dosage strength," might lead to BCS class migration, such as in the cases of metoclopramide and prednisone (2). Prof. Dr. Peter Langguth reviewed currently available in vitro (e.g., Caco-2 and MDCK cells monolayers), in situ (e.g., rat intestinal perfusion model), and in vivo (e.g., human intestinal perfusion model, absolute bioavailability, and mass balance) methods for assessing drug intestinal permeability. He cautioned about potential excipient effects on gastrointestinal motility as well as the possibility of them acting as competitors for some uptake and influx transporters, affecting drug absorption in a way that is not possible to anticipate by in vitro dissolution testing. This topic was further addressed by Prof. Dr. James Polli, who discussed the risks of extending BCS-based biowaiver decisions to BCS Class 3 drugs, focusing on potential excipient effects on drug absorption. He presented some recent unpublished data from his research team, showing that even though some 
common excipients did not seem to affect permeability of cimetidine and acyclovir, which are P-glycoprotein substrates, they can impact in vivo dissolution, as was observed after administering formulations containing high amounts of HPMC and magnesium stearate. However, as long as in vitro experimental conditions are biorelevant, excipient effects such as the latter ones described could be anticipated. He also discussed that in vitro studies might be better than traditional pharmacokinetic studies for assessing bioequivalence between test and reference formulations, highlighting the following advantages: (1) reduced costs; (2) in vitro dissolution tests directly assess product performance; (3) ethical considerations; and (4) elimination of problems arising from inconclusive in vivo bioequivalence studies showing high type II error. Prof. Dressman also spoke about FIP Biowaiver Monographs, a project that was and is mainly intended to publish Biowaiver Monographs of drug substances that appear on the WHO List of Essential Medicines and, occasionally, other drug substances finding very common use. The FIP Biowaiver Monograph reports all relevant data found in the open scientific literature and also reviews critically the reliability of these published scientific data and identifies gaps. So far, 43 monographs have been published, and many others are ongoing. Also, Prof. Dressman indicated that three of the ten most accessed articles in J. Pharm. Sci. in 2014 were FIP Biowaiver Monographs, highlighting the worldwide relevance of the project. Prof. Dr. Leslie Benet was the last speaker of the first session. His presentation was prerecorded since he had already accepted an invitation to attend another meeting on the same date. Prof. Benet explained that Biopharmaceutics Drug Disposition and Classification System (BDDCS) is a modification of BCS developed after identifying that there was a very good correlation between extent of drug absorption and extent of metabolism. He pointed out that there is no conflict between BCS and BDDCS but simply that each system has a different purpose and utilizes a different measure of intestinal permeability. While the objective of the BCS is to predict in vivo performance of drug products, BDDCS was proposed to serve as a basis for predicting the importance of transporters in determining drug bioavailability and disposition. In his opinion, any difference between BCS and BDDCS is not due to the systems per se, but to the ambiguous definition of permeability stated by FDA, which defines a kinetic parameter, intestinal permeability, in terms of a thermodynamic measure, extent of drug absorption.

In Plenary Session 2, Dr. Vinod Shah reviewed metrics currently available to compare dissolution profiles. These can be categorized as model-independent or model-dependent methods. He highlighted that the $f_{2}$ metric, initially proposed by Moore and Flanner (3) based on mean-squared difference and further statistically developed through mathematical scaling by Shah and coworkers $(4,5)$, ended up being the simplest and broadly accepted mathematical approach to compare dissolution profiles based on the assumption that an average difference of up to $10 \%$ between two dissolution profiles is not significant. Dr. Shah also emphasized that since the $f_{2}$ factor is calculated using mean percentage of drug dissolved at each sampling time point, high variability may bias the mean estimates. This can be overcome by using bootstrap methods (4) to build $90 \%$ confidence intervals. Nevertheless, standardization of simulation techniques in this field is still lacking. Prof. Dr. Bertil Abrahamson presented some experimental examples and suggestions of how clinical relevance of dissolution testing can be achieved in the context of Quality by Design (QbD), emphasizing that in vitro dissolution testing together with BCS considerations could provide a key link between manufacturing/product design variables and clinical safety/efficacy in QbD. Also, Prof. Dr. Abrahamson pointed out that IVIVR and physiologically based pharmacokinetic models (PBPK) can be useful tools to build "safe spaces" aiming to achieve regulatory flexibility, which in turn, may facilitate continuous improvement of both drug product and manufacturing process (6). Prof. Pablo Quiroga used the equation for calculating risk priority number (RPN) in Failure Mode and Effects Analysis (FMEA), first suggested by Kubinga and coworkers (7) as a useful tool for supporting biowaivers to assess the risks of extending BCS-based biowaiver decisions to BCS Class 2 drugs. He focused on the element of the risk equation related to the capacity of detection and reviewed the literature to identify whether the current set of experimental conditions recommended by BCS guidelines could anticipate the bioequivalence outcome for poorly soluble and highly absorbed drugs. Some issues related to the dynamic acidification of the thin diffusion layer in response to ionization of some weak acids, which in turn can affect the dissolution of the drug product itself, which do not seem to be captured by the high buffer capacity dissolution media were raised (8). This led to the conclusion that the current capacity of detection would need to be improved to allow a biowaiver for BCS Class 2 drugs. Prof. Peter Langguth was the last speaker of the afternoon session and spoke about predicting food effects on the oral drug product performance using BCS. He presented several examples, highlighting an influence of food on intestinal drug metabolism and uptake and efflux transport system and a negative food effect on BCS Class 3 drugs, mainly affecting drug substance (e.g., intestinal dilution) or drug product (e.g., increasing viscosity of dissolution fluid).

Plenary Session 3 was dedicated to the utilization of BCS principles in the regulatory environment. Six speakers presented a worldwide overview about BCS-based biowaiver requirements within different jurisdictions. Dr. Mehul Mehta presented the current decision tree that FDA has been using to evaluate BCS-based biowaiver submissions. He said that the Advisory BCS Committee has reviewed 63 submitted applications so far, and $67 \%$ of them were granted BCS-based biowaiver approval since 
they belonged to BCS Class 1. New drug and abbreviated drug applications accounted for $48 \%$ and $52 \%$ of the submissions, respectively. Dr. Mehta also emphasized that FDA has been reviewing its BCS guidance, and they will move toward accepting biowaiver applications for BCS Class 3 drugs in the future, but without changing the dose definition for solubility classification purposes. Prof. Dr. Dressman reviewed WHO, EMA, and FDA BCS guidances, pointing out the main differences among them. She discussed the European criteria that allow biowaivers for BCS Class 3 drugs. Although on one hand, the procedure is scientifically robust, on the other hand, it is unclear how regulatory authorities will handle the "quantitatively similar" criterion given that quantities of excipients in the reference formulation are not disclosed, except in some cases, in the Netherlands. Even though generic companies can use reverse engineering tools to unveil reference formulations, she pointed out that regulators will have to compare the product composition with the registered formulation. Some legal issues related to confidentiality may arise. Dr. Yanina Rodriguez spoke about the legal framework for generic drug registration in Argentina. She provided the audience with an overview of the possibilities of biowaivers accepted by the Administración Nacional de Medicamentos, Alimentos y Tecnología Médica (ANMAT), emphasizing biowaivers for lower dosage strengths in case of proportionality of the formulations and based on BCS principles, for some listed BCS Classes 1 and 3 drugs. Dr. Rodriguez mentioned that ANMAT performs some in vitro dissolution tests to assess whether the reference listed drugs will fulfill at least the rapid dissolution criterion before deciding to add a drug to the list. Dr. Gustavo Mendes Lima Santos spoke about the Brazilian legal framework for biowaivers, Resolução RDC n. 37/11. He presented the three possibilities of biowaiver currently available in Brazil: (1) self-evident bioequivalence; (2) lower dosage strengths; and (3) BCS based. Given that at the time of publication of the Brazilian BCS guideline, it was identified that the views about BCS principles were not adequately harmonized through academics, industrial scientists, and regulators, the Agência Nacional de Vigilância Sanitária (ANVISA) decided to start off with a stepwise approach, elaborating a dynamic list with some candidates for BCS-based biowaiver decisions. Dr. Santos explained that ANVISA and sponsors share responsibilities in this field. While ANVISA is responsible for the classification of the extent of drug absorbed, risk assessment in terms of drug therapeutic index, and evaluation of whether there is evidence of non-BE results not detected by in vitro dissolution tests for the selected drugs in its internal database (SINEB), sponsors should submit solubility and in vitro dissolution results according to the $B C S$ guideline. He also said that the Brazilian BCS guideline is currently under revision, and an update is expected by next year. Dr. Alexis Aceituno presented the Chilean legal framework for bioequivalence and biowaiver. He said that initially, the Instituto de Salud
Publica (ISP) required in vivo BE studies only for two drugs, but currently, the number of drugs has increased to more than 150. He highlighted that BCS-based biowaivers can be granted for some BCS Classes 1, 2, and 3 in a case-by-case approach. The last speaker of this session was Dr. Mariana Pagano, who represented the Ministry of Health of Uruguay. She discussed the main points of the legal framework within her jurisdiction related to interchangeability of generics, mentioning that $\mathrm{BCS}$ principles had already been incorporated into the Uruguayan guidelines. At the end of this session, it was possible to identify some divergences among the BCS guidelines issued by South American regulatory authorities and also among FDA, EMA, and WHO. A harmonization of such criteria would facilitate future mutual recognition and information exchange within the regulatory environment. Thus, any attempt to harmonize dose definition for solubility classification purposes, cutoff value for classifying the extent of drug absorption, experimental in vitro dissolution conditions (e.g., for 50 or 75 rpm for paddle apparatus?), and BCS-based biowaiver extensions beyond Class 1 drugs is very welcome.

Plenary Session 4 was dedicated to in silico methods. Prof. Dr. Bertil Abrahamson spoke about the Oral Biopharmaceutics Tools (OrBiTo) Project showing the four Work Packages (9-11). OrBiTo is a partnership among pharmaceutical companies, universities, and commercial PBPK software development companies, whose main goal is to improve tools to predict the performance of orally administered drugs. Prof. Abrahamson discussed the current situation of the project and the main issues (e.g., lack of scientific understanding in areas like intestinal precipitation, gastrointestinal hydrolysis, and differences in regional permeability). He also highlighted that conventional quality control dissolution methods often fail to predict in vivo outcomes, and sometimes they are overdiscriminative to variables that are not relevant in vivo. Prof. Dr. Alan Telavi discussed the importance of having in silico models to predict drug intestinal permeability. Prof. Dr. Telavi spoke about some efforts aimed at the integrated prediction of several significant molecular properties in the field of drug discovery, such as pharmacological activity, aqueous solubility, human intestinal permeability, and affinity to P-glycoprotein. Dr. Rodrigo Cristofoletti discussed the importance of taking PK/PD relationships into consideration to set clinically relevant limits for in vivo bioequivalence studies (12), according to the scientific background provided by the Biopharmaceutics Risk Assessment Roadmap (BIORAM) (13). He also emphasized the importance of in silico tools like PBPK/PD models to explore "what if" scenarios, and sensitivity analysis that may be useful to identify the most relevant variables, which in turn should be more thoroughly investigated using in vitro or in vivo methods. Dr. Cristofoletti concluded that such approaches may help in building clinically relevant "safe spaces" that can facilitate continuous improvement of the formulation based on science and risk-based regulatory 
flexibility. Prof. Dressman returned to the stage one more time to speak about using in vitro results to predict in vivo outcomes with PBPK models as convolution techniques. She highlighted the importance of using biorelevant experimental conditions when trying to predict in vivo dissolution. Prof. Dr. Dressman also provided the audience with some successful examples of in vitro-in silico-in vivo extrapolation (IV-IS-IV-E) for poorly soluble drugs like aprepitant and nifedipine $(14,15)$.

\section{ACKNOWLEDGMENTS}

On behalf of the Organizing Committee, we would like to thank all the speakers and moderators who participated in the workshop as well as the FIP and AAPS for financial support.

\section{REFERENCES}

1. Amidon, G. L.; Lennernäs, H.; Shah, V. P.; Crison, J. R. A Theoretical Basis for a Biopharmaceutic Drug Classification: The Correlation of in Vitro Drug Product Dissolution and in Vivo Bioavailability. Pharm. Res. 1995, 12 (3), 413-420. DOI: 10.1023/A:1016212804288.

2. Sediq, A.; Kubbinga, M.; Langguth, P.; Dressman, J. The Impact of the EMA Change in Definition of "Dose" on the BCS Dose-Solubility Ratio: A Review of the Biowaiver Monographs. J. Pharm. Sci. 2014, 103 (1), 65-70. DOI: 10.1002/jps.23769.

3. Moore, J. W.; Flanner, H. H. Mathematical Comparison of Curves with an Emphasis on In-Vitro Dissolution Profiles. Pharm. Technol. 1996, 20 (6), 64-74.

4. Shah, V. P.; Tsong, Y.; Sathe, P.; Liu, J. P. In Vitro Dissolution Profile Comparison-Statistics and Analysis of the Similarity Factor, $f_{2}$. Pharm. Res. 1998, 15 (6), 889-896. DOI: 10.1023/A:1011976615750.

5. Shah, V. P.; Tsong, Y.; Sathe, P.; Williams, R. L. Dissolution Profile Comparison Using Similarity Factor, f2. Dissolution Technol. 1999, 6 (3), 15.

6. Dickinson, P. A.; Lee, W. W.; Stott, P. W.; Townsend, A. I.; Smart, J. P.; Ghahramani, P.; Hammett, T.; Billett, L.; Behn, S.; Gibb, R. C.; Abrahamsson, B. Clinical Relevance of Dissolution Testing in Quality by Design. AAPS J. 2008, 10 (2), 380-390. DOI: 10.1208/s12248-008-9034-7.

7. Kubbinga, M.; Langguth, P.; Barends, D. Risk analysis in bioequivalence and biowaiver decisions. Biopharm. Drug Dispos. 2013, 34 (5), 254-261. DOI: 10.1002/ bdd.1831.

8. Tsume, Y.; Langguth, P.; Garcia-Arieta, A.; Amidon, G. L. In silico prediction of drug dissolution and absorption with variation in intestinal $\mathrm{pH}$ for BCS class II weak acid drugs: ibuprofen and ketoprofen. Biopharm. Drug Dispos. 2012, 33 (7), 366-377. DOI: 10.1002/bdd.1800.

9. Sjögren, E.; Abrahamsson, B.; Augustijns, P.; Becker,
D.; Bolger, M. B.; Brewster, M.; Brouwers, J.; Flanagan, T.; Harwood, M.; Heinen, C.; Holm, R.; Juretschke, H. P.; Kubbinga, M.; Lindahl, A.; Lukacova, V.; Münster, U.; Neuhoff, S.; Nguyen, M. A.; Peer, A.; Reppas, C.; Hodjegan, A. R.; Tannergren, C.; Weitschies, W.; Wilson, C.; Zane, P.; Lennernäs, H.; Langguth, P. In vivo methods for drug absorption-Comparative physiologies, model selection, correlations with in vitro methods (IVIVC), and applications for formulation/API/excipient characterization including food effects. Eur. J. Pharm. Sci. 2014, 57, 99-151. DOI: 10.1016/j.ejps.2014.02.010.

10. Bergström, C. A.; Holm, R.; Jørgensen, S. A.; Andersson, S. B.; Artursson, P.; Beato, S.; Borde, A.; Box, K.; Brewster, M.; Dressman, J.; Feng, K. I.; Halbert, G.; Kostewicz, E.; McAllister, M.; Muenster, U.; Thinnes, J.; Taylor, R.; Mullertz, A. Early pharmaceutical profiling to predict oral drug absorption: Current status and unmet needs. Eur. J. Pharm. Sci. 2014, 57, 173-199. DOI: 10.1016/j. ejps.2013.10.015.

11. Kostewicz, E. S.; Aarons, L.; Bergstrand, M.; Bolger, M. B.; Galetin, A.; Hatley, O.; Jamei, M.; Lloyd, R.; Pepin, X.; Rostami-Hodjegan, A.; Sjögren, E.; Tannergren, C.; Turner, D. B.; Wagner, C.; Weitschies, W.; Dressman, J. PBPK models for the prediction of in vivo performance of oral dosage forms. Eur. J. Pharm. Sci. 2014, 57, 300 321. DOI: 10.1016/j.ejps.2013.09.008.

12. Cristofoletti, R.; Dressman, J. B. Use of Physiologically Based Pharmacokinetic Models Coupled with Pharmacodynamic Models to Assess the Clinical Relevance of Current Bioequivalence Criteria for Generic Drug Products Containing Ibuprofen. J. Pharm. Sci. 2014, 103 (10), 3263-3275. DOI: 10.1002/jps.24076.

13. Selen, A.; Dickinson, P. A.; Müllertz, A.; Crison, J. R.; Mistry, H. B.; Cruañes, M. T.; Martinez, M. N.; Lennernäs, H.; Wigal, T. L.; Swinney, D. C.; Polli, J. E.; Serajuddin, A.T.; Cook, J. A.; Dressman, J. B. The Biopharmaceutics Risk Assessment Roadmap for Optimizing Clinical Drug Product Performance. J. Pharm. Sci. 2014, 103 (11), 3377-3397. DOI: 10.1002/jps.24162.

14. Shono, Y.; Jantratid, E.; Kesisoglou, F.; Reppas, C.; Dressman, J. B. Forecasting in vivo oral absorption and food effect of micronized and nanosized aprepitant formulations in humans. Eur. J. Pharm. Biopharm. 2010, 76 (1), 95-104. DOI: 10.1016/j.ejpb.2010.05.009.

15. Wagner, C.; Thelen, K.; Willmann, S.; Selen, A.; Dressman, J. B. Utilizing In Vitro and PBPK Tools to Link ADME Characteristics to Plasma Profiles: Case Example Nifedipine Immediate Release Formulation. J. Pharm. Sci. 2013, 102 (9), 3205-3219. DOI: 10.1002/jps.23611. 


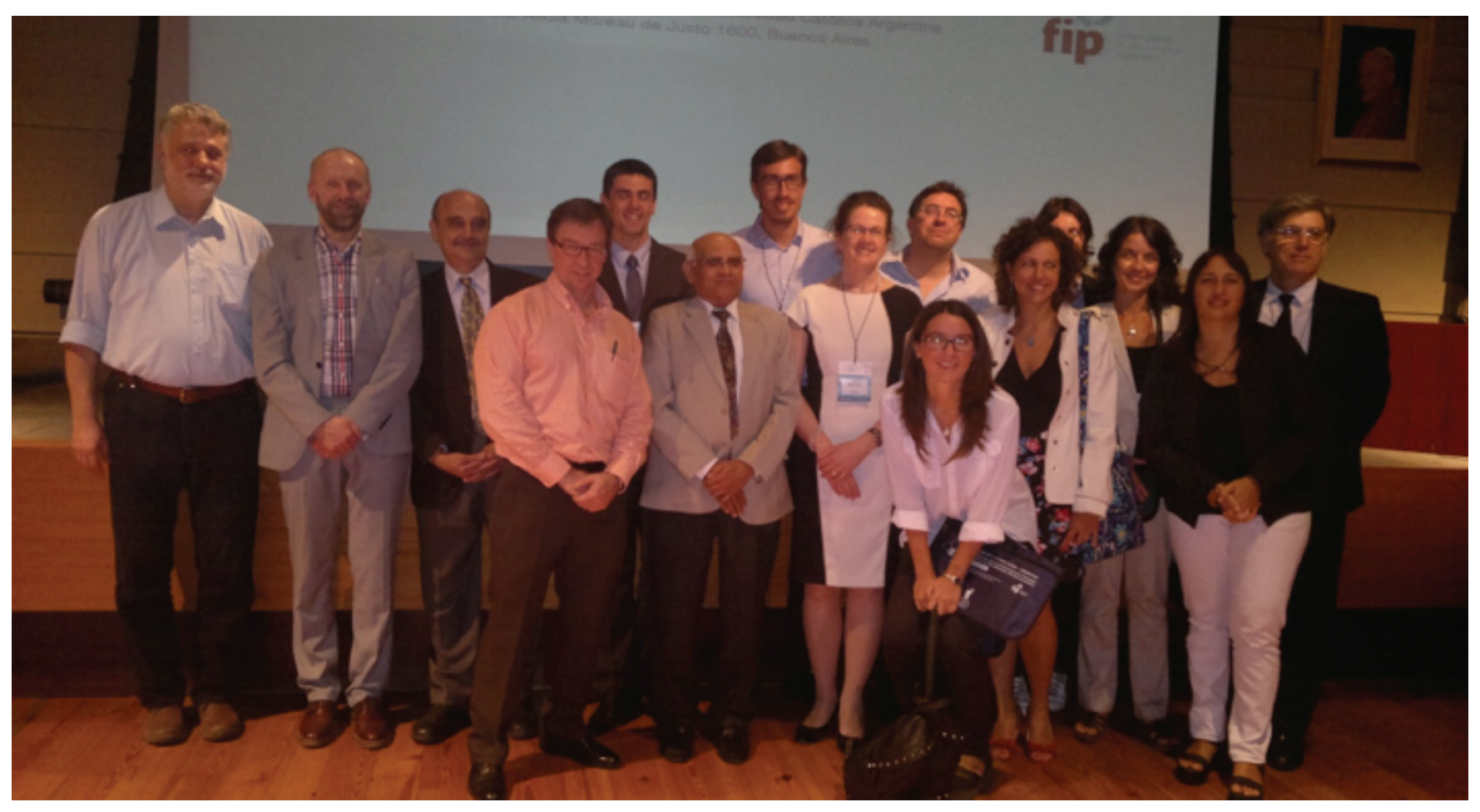

Workshop speakers and moderators (left to right). Back row: Prof. Dr. Peter Langguth, Prof. Dr. Bertil Abrahamson, Dr.Mehul Mehta, Dr. Rodrigo Cristofoletti, Dr. Gustavo Mendes Lima Santos, Prof. Pablo Quiroga, Prof. Dr. Alan Telavi, Dr. Mariana Pagano, and Prof. Arturo Hoya. Front row: Prof. Dr. James Polli, Dr. Vinod Shah, Prof. Dr. Jennifer Dressman, Prof. Dr. Maria Esperanza Ruiz, Dr. Yanina Rodriguez, and Prof. Dr. Silvia Lucangioli. 